\title{
Dense Quark Matter in a Magnetic Field
}

\author{
Efrain J. Ferrer, Vivian de la Incera* \\ Western Illinois University, USA \\ E-mail: ej-ferrer@wiu.edu, v-incera@wiu.edu \\ Cristina Manuel \\ Instituto de Fisica Corpuscular (CSIC-U. de Valencia), Spain \\ E-mail: Cristina.Manueleific.uv.es
}

We explore the effects of an applied strong external magnetic field in the structure and magnitude of the color superconducting diquark condensate of a three massless flavor theory. The long-range component of the B field that penetrates the superconductor enhances the condensates formed by quarks charged with respect to this electromagnetic field.

29th Johns Hopkins Workshop in Theoretical Physics: Strong Matter in the Heavens. Budapest, August 1-3, 2005

${ }^{*}$ Speaker. 


\section{Introduction}

It is well established that at high baryon density the combination of asymptotic freedom and the existence of attractive channels in the color interaction between the quarks lying in the large Fermi surface come together to promote the formation of quark-quark pairs, which in turn break the color gauge symmetry giving rise to the phenomenon of color superconductivity. At densities much higher than the masses of the $\mathrm{u}, \mathrm{d}$, and s quarks, one can assume the three quarks as massless and the favored state results to be the so-called Color-Flavor-Locking (CFL) phase [1], characterized by a spin zero diquark condensate antisymmetric in both color and flavor.

The conditions of extremely large density and very low temperature required for color superconductivity cannot be recreated in Earth's labs. Fortunately, nature provides us with a laboratory to probe color superconductivity, the cores of celestial compact objects. These compact stars typically have very large magnetic fields. Neutron stars can have magnetic fields as large as $B \sim 10^{12}-10^{14}$ $\mathrm{G}$ in their surfaces, while in magnetars they are in the range $B \sim 10^{14}-10^{15} \mathrm{G}$, and perhaps as high as $10^{16} \mathrm{G}$ [2] (for a recent review of magnetic fields in dense stars see [3]). Even though we do not know yet of any suitable mechanism to produce more intense fields, the virial theorem [4] allows the field magnitude to reach values as large as $10^{18}-10^{19} \mathrm{G}$. If quark stars are self-bound rather than gravitational-bound objects, the upper limit that has been obtained by comparing the magnetic and gravitational energies, could go even higher.

A natural question to ask is: What is the effect, if any, of the huge star's magnetic field in the color superconducting core? A complete answer to this question would require a rather involved study of quark matter at the intermediate range of densities proper of neutron stars, where the strange quark mass cannot be ignored, with the additional complication of an extra parameter, the magnetic field. However, as a first, more tractable approach to this question, one can ignore the strange quark mass effects and look for the consequences of an external magnetic field on the superconducting phase, assuming that the quark matter is formed by three massless flavors. This was the strategy followed in our recent paper [5], whose main results will be described in what follows.

In this talk I will show the way a magnetic field affects the pairing structure and hence its symmetry, ultimately producing a different superconducting phase that we have called Magnetic Color-Flavor-Locking (MCFL) phase.

In a conventional superconductor, since Cooper pairs are electrically charged, the electromagnetic gauge invariance is spontaneously broken, thus the photon acquires a Meissner mass that can screen a weak magnetic field, the phenomenon of Meissner effect. In spin-zero color superconductivity, although the color condensate has non-zero electric charge, there is a linear combination of the photon and a gluon that remains massless [1]. This new field plays the role of the "in-medium" photon in the color superconductor, so the propagation of light in the color superconductor is different from that in an electric superconductor.

Because of the long-range "rotated" electromagnetic field, a spin-zero color superconductor may be penetrated by a rotated magnetic field $\widetilde{B}$. Although a few works [6] had previously addressed the problem of the interaction of an external magnetic field with dense quark matter, none of these studies considered the modification produced by the field on the gap itself. However, as we have recently shown [5], the gap structure gets modified due to the penetrating field. To un- 
derstand this, notice that, although the condensate is $\widetilde{Q}$-neutral, some of the quarks participating in the pairing are $\widetilde{Q}$-charged and hence can couple to the background field, which in turn affects the gap equations through the Green functions of these $\widetilde{Q}$-charged quarks. Due to the coupling of the charged quarks with the external field, the color-flavor space is augmented by the $\widetilde{Q}$-charge color-flavor operator, and consequently the CFL order parameter splits in new independent pieces giving rise to a new phase, the MCFL phase.

\section{The MCFL Gap Structure}

The linear combination of the photon $A_{\mu}$ and a gluon $G_{\mu}^{8}$ that behaves as a long-range field in the spin-zero color superconductor is given by $[1,7]$,

$$
\widetilde{A}_{\mu}=\cos \theta A_{\mu}-\sin \theta G_{\mu}^{8}
$$

while the orthogonal combination $\widetilde{G}_{\mu}^{8}=\sin \theta A_{\mu}+\cos \theta G_{\mu}^{8}$ is massive. In the CFL phase the mixing angle $\theta$ is sufficiently small ( $\sin \theta \sim e / g \sim 1 / 40$ ). Thus, the penetrating field in the color superconductor is mostly formed by the photon with only a small gluon admixture.

The unbroken $U(1)$ group corresponding to the long-range rotated photon (i.e. the $\widetilde{U}(1)_{\text {e.m. }}$ ) is generated, in flavor-color space, by $\widetilde{Q}=Q \times 1-1 \times Q$, where $Q$ is the electromagnetic charge generator. We use the conventions $Q=-\lambda_{8} / \sqrt{3}$, where $\lambda_{8}$ is the 8 th Gell-Mann matrix. Thus our flavor-space ordering is $(s, d, u)$. In the 9-dimensional flavor-color representation that we will use in this paper (the color indexes we are using are $(1,2,3)=(\mathrm{b}, \mathrm{g}, \mathrm{r}))$, the $\widetilde{Q}$ charges of the different quarks, in units of $\widetilde{e}=e \cos \theta$, are

\begin{tabular}{|c|c|c|c|c|c|c|c|c|}
\hline$s_{1}$ & $s_{2}$ & $s_{3}$ & $d_{1}$ & $d_{2}$ & $d_{3}$ & $u_{1}$ & $u_{2}$ & $u_{3}$ \\
\hline 0 & 0 & - & 0 & 0 & - & + & + & 0 \\
\hline
\end{tabular}

In the presence of an external rotated magnetic field the kinetic part of the quarks' Lagrangian density must be rewritten using the covariant derivative

$$
L_{q u a r k s}^{e m}=\bar{\psi}\left(i \Pi_{\mu} \gamma^{\mu}\right) \psi
$$

with

$$
\Pi_{\mu}=i \partial_{\mu}+\widetilde{e} \widetilde{Q} \widetilde{A}_{\mu}
$$

where

$$
\widetilde{Q}=\Omega_{+}-\Omega_{-} .
$$

is the rotated charge operator. The charge projectors

$$
\begin{aligned}
& \Omega_{+}=\operatorname{diag}(0,0,0,0,0,0,1,1,0), \\
& \Omega_{-}=\operatorname{diag}(0,0,1,0,0,1,0,0,0),
\end{aligned}
$$


and

$$
\Omega_{0}=\operatorname{diag}(1,1,0,1,1,0,0,0,1),
$$

obey the algebra

$$
\begin{gathered}
\Omega_{\eta} \Omega_{\eta^{\prime}}=\delta_{\eta \eta^{\prime}} \Omega_{\eta}, \quad \eta, \eta^{\prime}=0,+,-. \\
\Omega_{0}+\Omega_{+}+\Omega_{-}=1 .
\end{gathered}
$$

The rotated magnetic field naturally separates the quark fields according to their $\tilde{Q}$ charge. The fermion field in the $9 \times 9$ representation used above, $\psi^{T}=\left(s_{1}, s_{2}, s_{3}, d_{1}, d_{2}, d_{3}, u_{1}, u_{2}, u_{3}\right)$, can then be written as the sum of three fields with zero, positive and negative rotated electromagnetic charges,

$$
\psi=\psi_{(0)}+\psi_{(+)}+\psi_{(-)}
$$

where the $(0)-,(+/-)$-charged fields can be respectively written in terms of the charge projectors as

$$
\psi_{(0)}=\Omega_{0} \psi, \quad \psi_{(+)}=\Omega_{+} \psi, \quad \psi_{(-)}=\Omega_{-} \psi .
$$

A strong magnetic field affects the flavor symmetries of $\mathrm{QCD}$, as different quark flavors have different electromagnetic charges. For three light quark flavors, only the subgroup of $S U(3)_{L} \times$ $S U(3)_{R}$ that commutes with $Q$, the electromagnetic generator, is a symmetry of the theory. Similarly, in the CFL phase a strong $\widetilde{B}$ field should affect the symmetries of the theory, as $\widetilde{Q}$ does not commute with the whole locked $S U(3)$ group. Based on the above considerations, and imposing that the condensate should retain the highest degree of symmetry, we proposed [5] the following ansatz for the gap structure in the presence of a magnetic field

$$
\Delta=\left(\begin{array}{ccccccccc}
2 \Delta_{S}^{\prime} & 0 & 0 & 0 & \Delta_{A}+\Delta_{S} & 0 & 0 & 0 & \Delta_{A}^{B}+\Delta_{S}^{B} \\
0 & 0 & 0 & \Delta_{S}-\Delta_{A} & 0 & 0 & 0 & 0 & 0 \\
0 & 0 & 0 & 0 & 0 & 0 & \Delta_{S}^{B}-\Delta_{A}^{B} & 0 & 0 \\
0 & \Delta_{S}-\Delta_{A} & 0 & 0 & 0 & 0 & 0 & 0 & 0 \\
\Delta_{A}+\Delta_{S} & 0 & 0 & 0 & 2 \Delta_{S}^{\prime} & 0 & 0 & 0 & \Delta_{A}^{B}+\Delta_{S}^{B} \\
0 & 0 & 0 & 0 & 0 & 0 & 0 & \Delta_{S}^{B}-\Delta_{A}^{B} & 0 \\
0 & 0 & \Delta_{S}^{B}-\Delta_{A}^{B} & 0 & 0 & 0 & 0 & 0 & 0 \\
0 & 0 & 0 & 0 & 0 & \Delta_{S}^{B}-\Delta_{A}^{B} & 0 & 0 & 0 \\
\Delta_{A}^{B}+\Delta_{S}^{B} & 0 & 0 & 0 & \Delta_{A}^{B}+\Delta_{S}^{B} & 0 & 0 & 0 & 2 \Delta_{S}^{\prime \prime}
\end{array}\right)
$$

We call the reader's attention to the fact that despite the $\widetilde{Q}$-neutrality of all the condensates, they can be composed either by neutral or by charged quarks. Condensates formed by $\widetilde{Q}$-charged quarks feel the field directly through the minimal coupling of the background field $\widetilde{B}$ with the quarks in the pair. A subset of the condensates formed by $\widetilde{Q}$-neutral quarks, can feel the presence of the field via tree-level vertices that couple them to charged quarks. The gaps $\Delta_{A / S}^{B}$ are antisymmetric/symmetric combinations of condensates composed by charged quarks and condensates 
formed by this kind of neutral quarks. The gaps $\Delta_{A}$, as well as $\Delta_{S}, \Delta_{S}^{\prime}$ and $\Delta_{S}^{\prime \prime}$, on the other hand, are antisymmetric and symmetric combinations of condensates formed by neutral quarks that do not belong to the above subset. The only way the field can affect them is through the system of highly non-linear coupled gap equations. At zero field the CFL gap matrix is recovered since in that case $\Delta_{A}^{B}=\Delta_{A}$ and $\Delta_{S}^{B}=\Delta_{S}=\Delta_{S}^{\prime}=\Delta_{S}^{\prime \prime}$.

Although the symmetry of the problem allows for four independent symmetric gaps, the condensates $\Delta_{S}^{\prime}$ and $\Delta_{S}^{\prime \prime}$ are only due to subleading color symmetric interactions, and as explained in the previous paragraph, they are formed by neutral quarks that are not coupled to charged quarks, so they belong to the same class as $\Delta_{S}$. Therefore, there is no reason to expect that they will differ much from $\Delta_{S}$. Hence, in a first approach to the problem, we will consider $\Delta_{S} \simeq \Delta_{S}^{\prime} \simeq \Delta_{S}^{\prime \prime}$.

The order parameter (2.13) implies the following symmetry breaking pattern: $S U(3)_{\text {color }} \times$ $S U(2)_{L} \times S U(2)_{R} \times U(1)_{B} \times U^{(-)}(1)_{A} \times U(1)_{\text {e.m. }} \rightarrow S U(2)_{\text {color }+L+R} \times \widetilde{U}(1)_{\text {e.m. }}$. The $U^{(-)}(1)_{A}$ symmetry is connected with the current which is an anomaly-free linear combination of $s, d$ and $u$ axial currents [8]. The locked $S U(2)$ corresponds to the maximal unbroken symmetry, and as such it maximizes the condensation energy. Notice that it commutes with the rotated electromagnetic group $\widetilde{U}(1)_{\text {e.m. }}$.

The counting of broken generators, after taking into account the Anderson-Higgs mechanism, tells us that there are only five Nambu-Goldstone bosons. One is associated to the breaking of the baryon symmetry; three Goldstone bosons are associated to the breaking of $S U(2)_{A}$, and another one associated to the breaking of $U^{(-)}(1)_{A}$. All the Nambu-Goldstone bosons are $\widetilde{Q}$-neutral. The number and properties of the lightest particles in the MCFL have implications for the low-energy physics of the phase. Since in her talk Cristina Manuel will address the low-energy physics of the MCFL phase, I will not extend on this topic in mine.

\section{Effective Action in the Presence of a Magnetic Field}

Let us construct the effective action of the system in the presence of a magnetic field. With this aim, we will use a Nambu-Jona-Lasinio (NJL) four-fermion interaction abstracted from one-gluon exchange [1]. Although this simplified treatment disregards the effect of the $\widetilde{B}$-field on the gluon dynamics and assumes the same NJL couplings for both the situation with and without magnetic field, it keeps the main attributes of the theory, thereby providing the correct qualitative physics.

We start from the mean-field effective action

$$
\begin{array}{r}
I_{B}(\bar{\psi}, \psi)=\int_{x, y}\left\{\frac { 1 } { 2 } \left[\bar{\psi}_{(0)}(x)\left[G_{(0) 0}^{+}\right]^{-1}(x, y) \psi_{(0)}(y)+\bar{\psi}_{(+)}(x)\left[G_{(+) 0}^{+}\right]^{-1}(x, y) \psi_{(+)}(y)\right.\right. \\
+\bar{\psi}_{(-)}(x)\left[G_{(-) 0}^{+}\right]^{-1}(x, y) \psi_{(-)}(y)+\bar{\psi}_{(0) C}(x)\left[G_{(0) 0}^{-}\right]^{-1}(x, y) \psi_{(0) C}(y) \\
\left.+\bar{\psi}_{(+) C}(x)\left[G_{(+) 0}^{-}\right]^{-1}(x, y) \psi_{(+) C}(y)+\bar{\psi}_{(-) C}(x)\left[G_{(-) 0}^{-}\right]^{-1}(x, y) \psi_{(-) C}(y)\right] \\
+\frac{1}{2}\left[\bar{\psi}_{(0) C}(x) \Delta^{+}(x, y) \psi_{(0)}(y)+\text { h.c. }\right]+\frac{1}{2}\left[\bar{\psi}_{(+) C}(x) \Delta^{+}(x, y) \psi_{(-)}(y)\right. \\
\left.\left.+\bar{\psi}_{(-) C}(x) \Delta^{+}(x, y) \psi_{(+)}(y)+h . c .\right]\right\}
\end{array}
$$

where the external magnetic field has been explicitly introduced through minimal coupling with the $\widetilde{Q}$-charged fermions. The presence of the field is also taken into account in the diquark condensate $\Delta^{+}=\gamma_{5} \Delta$, whose color-flavor structure is given by Eq.(2.13). 
In (3.1) symbols in parentheses indicate neutral $(0)$, positive $(+)$ or negative $(-) \tilde{Q}$-charged quarks. Supra-indexes + or - in the propagators indicate, as it is customary, whether it is the inverse propagator of a field or conjugated field respectively. Then, for example, $\left[G_{(+) 0}^{-}\right]^{-1}$ corresponds to the bare inverse propagator of positively charged conjugate fields, and so on. The explicit expressions of the inverse propagators are

$$
\begin{gathered}
{\left[G_{(0) 0}^{ \pm}\right]^{-1}(x, y)=\left[i \gamma^{\mu} \partial_{\mu}-m \pm \mu \gamma^{0}\right] \delta^{4}(x-y),} \\
{\left[G_{(+) 0}^{ \pm}\right]^{-1}(x, y)=\left[i \gamma^{\mu} \Pi_{\mu}^{(+)}-m \pm \mu \gamma^{0}\right] \delta^{4}(x-y),} \\
{\left[G_{(-) 0}^{ \pm}\right]^{-1}(x, y)=\left[i \gamma^{\mu} \Pi_{\mu}^{(-)}-m \pm \mu \gamma^{0}\right] \delta^{4}(x-y),}
\end{gathered}
$$

with

$$
\Pi_{\mu}^{( \pm)}=i \partial_{\mu} \pm \widetilde{e} \widetilde{A}_{\mu}
$$

Transforming the field-dependent quark propagators to momentum space can be performed with the use of the Ritus' method, originally developed for charged fermions [9] and recently extended to charged vector fields [10]. In Ritus' approach the diagonalization in momentum space of charged fermion Green's functions in the presence of a background magnetic field is carried out using the eigenfunction matrices $E_{p}(x)$. These are the wave functions of the asymptotic states of charged fermions in a uniform magnetic field and play the role in the magnetized medium of the usual plane-wave (Fourier) functions $e^{i p x}$ at zero field. Below we introduce the basic properties of this transformation.

The transformation functions $E_{q}^{( \pm)}(x)$ for positively $(+)$, and negatively (-) charged fermion fields are obtained as the solutions of the field dependent eigenvalue equation

$$
\left(\Pi^{( \pm)} \cdot \gamma\right) E_{q}^{( \pm)}(x)=E_{q}^{( \pm)}(x)\left(\gamma \cdot \bar{p}^{( \pm)}\right)
$$

with $\bar{p}^{( \pm)}$given by

$$
\bar{p}^{( \pm)}=\left(p_{0}, 0, \pm \sqrt{2|\widetilde{e} \widetilde{B}| k,} p_{3}\right)
$$

and

$$
E_{q}^{( \pm)}(x)=\sum_{\sigma} E_{q \sigma}^{( \pm)}(x) \delta(\sigma)
$$

with eigenfunctions

$$
E_{p \sigma}^{( \pm)}(x)=\mathscr{N}_{n_{( \pm)}} e^{-i\left(p_{0} x^{0}+p_{2} x^{2}+p_{3} x^{3}\right)} D_{n_{( \pm)}}\left(\rho_{( \pm)}\right),
$$

where $D_{n_{( \pm)}}\left(\rho_{( \pm)}\right)$are the parabolic cylinder functions with argument $\rho_{( \pm)}$defined by

$$
\rho_{( \pm)}=\sqrt{2|\widetilde{e} \widetilde{B}|}\left(x_{1} \pm p_{2} / \widetilde{e} \widetilde{B}\right)
$$


and index $n_{( \pm)}$given by

$$
n_{( \pm)} \equiv n_{( \pm)}(k, \sigma)=k \pm \frac{\widetilde{e} \widetilde{B}}{2|\widetilde{e} \widetilde{B}|} \sigma-\frac{1}{2}, \quad n_{( \pm)}=0,1,2, \ldots
$$

$k=0,1,2,3, \ldots$ is the Landau level, and $\sigma$ is the spin projection that can take values \pm 1 only. Notice that in the lowest Landau level, $k=0$, only particles with one of the two spin projections, namely, $\sigma=1$ for positively charged particles, are allowed. The normalization constant $\mathscr{N}_{n_{( \pm)}}$is

$$
\mathscr{N}_{n_{( \pm)}}=(4 \pi|\widetilde{e} \widetilde{B}|)^{\frac{1}{4}} / \sqrt{n_{( \pm)} !} .
$$

In (3.8) the spin matrices $\delta(\sigma)$ are defined as

$$
\delta(\sigma)=\operatorname{diag}\left(\delta_{\sigma 1}, \delta_{\sigma-1}, \delta_{\sigma 1}, \delta_{\sigma-1}\right), \quad \sigma= \pm 1,
$$

and satisfy the following relations

$$
\begin{gathered}
\delta( \pm)^{\dagger}=\delta( \pm), \quad \delta( \pm) \delta( \pm)=\delta( \pm), \quad \delta( \pm) \delta(\mp)=0, \\
\gamma^{\prime} \delta( \pm)=\delta( \pm) \gamma^{\prime \prime}, \quad \gamma^{\perp} \delta( \pm)=\delta(\mp) \gamma^{\perp} .
\end{gathered}
$$

In Eq. (3.15) the notation $\gamma^{\prime \prime}=\left(\gamma^{0}, \gamma^{3}\right)$ and $\gamma^{\perp}=\left(\gamma^{1}, \gamma^{2}\right)$ was used.

The functions $E_{p}^{( \pm)}$are complete

$$
\sum_{k} \int d p_{0} d p_{2} d p_{3} E_{p}^{( \pm)}(x) \bar{E}_{p}^{( \pm)}(y)=(2 \pi)^{4} \delta^{(4)}(x-y)
$$

and orthonormal,

$$
\int_{x} \bar{E}_{p^{\prime}}^{( \pm)}(x) E_{p}^{( \pm)}(x)=(2 \pi)^{4} \Lambda_{k} \delta_{k k^{\prime}} \delta\left(p_{0}-p_{0}^{\prime}\right) \boldsymbol{\delta}\left(p_{2}-p_{2}^{\prime}\right) \boldsymbol{\delta}\left(p_{3}-p_{3}^{\prime}\right)
$$

with the $(4 \times 4)$ matrix $\Lambda_{k}$ given by

$$
\Lambda_{k}=\left\{\begin{array}{cll}
\delta(\sigma=\operatorname{sgn}[e B]) & \text { for } & k=0, \\
I & \text { for } & k>0 .
\end{array}\right.
$$

The matrix structure $\Lambda_{k}$ was recently introduced in Ref. [11]. It had been previously omitted in the orthonormal condition of the $E_{p}(x)$ functions given in Refs. [9, 10, 12]. Nevertheless, it should be underlined that this matrix only appears in the zero Landau level contribution, and consequently it enters as an irrelevant multiplicative factor in the Schwinger-Dyson equations in the lowest Landau level approximation. Thus, all the results obtained in the works $[9,10,12]$ remain valid. In Eqs. (3.16)-(3.17) we introduced the notation $\bar{E}_{p}^{( \pm)}(x)=\gamma_{0}\left(E_{p}^{( \pm)}(x)\right)^{\dagger} \gamma_{0}$.

Under the $E_{p}(x)$ functions, positively $\left(\psi_{(+)}\right)$, negatively $\left(\psi_{(-)}\right)$charged fields transform according to

$$
\psi_{( \pm)}(x)=\sum_{k} \int d p_{0} d p_{2} d p_{3} E_{p}^{( \pm)}(x) \psi_{( \pm)}(p)
$$




$$
\bar{\psi}_{( \pm)}(x)=\sum_{k} \int d p_{0} d p_{2} d p_{3} \bar{\psi}_{( \pm)}(p) \bar{E}_{p}^{( \pm)}(x) .
$$

One can show that

$$
\left[\gamma_{\mu}\left(\Pi_{(+) \mu} \pm \mu \delta_{\mu 0}\right)-m\right] E_{p}^{(+)}(x)=E_{p}^{(+)}(x)\left[\gamma_{\mu}\left(\bar{p}_{\mu}^{(+)} \pm \mu \delta_{\mu 0}\right)-m\right],
$$

and

$$
\left[\gamma_{\mu}\left(\Pi_{(-) \mu} \pm \mu \delta_{\mu 0}\right)-m\right] E_{p}^{(-)}(x)=E_{p}^{(-)}(x)\left[\gamma_{\mu}\left(\bar{p}_{\mu}^{(-)} \pm \mu \delta_{\mu 0}\right)-m\right] .
$$

The conjugate fields transform according to,

$$
\begin{aligned}
& \psi_{(+) C}(x)=\sum_{k} \int d p_{0} d p_{2} d p_{3} E_{p}^{(-)}(x) \psi_{(+) C}(p), \\
& \psi_{(-) C}(x)=\sum_{k} \int d p_{0} d p_{2} d p_{3} E_{p}^{(+)}(x) \psi_{(-) C}(p) .
\end{aligned}
$$

After transforming to momentum space one can introduce Nambu-Gorkov fermion fields of different $\tilde{Q}$ charges. They are the $\tilde{Q}$-neutral Gorkov field

$$
\Psi_{(0)}=\left(\begin{array}{c}
\psi_{(0)} \\
\psi_{(0) C}
\end{array}\right),
$$

the positive

$$
\Psi_{(+)}=\left(\begin{array}{c}
\psi_{(+)} \\
\psi_{(-) C}
\end{array}\right)
$$

and the negative one

$$
\Psi_{(-)}=\left(\begin{array}{c}
\psi_{(-)} \\
\psi_{(+) C}
\end{array}\right) .
$$

Using them, the Nambu-Gorkov effective action in the presence of a constant magnetic field $\widetilde{B}$ can be written as

$$
\begin{array}{r}
I^{B}(\bar{\psi}, \psi)=\frac{1}{2} \int \frac{d^{4} p}{(2 \pi)^{4}} \bar{\Psi}_{(0)}(p) \mathscr{S}_{(0)}^{-1}(p) \Psi_{(0)}(p) \\
+\frac{1}{2} \int \frac{d^{4} p}{(2 \pi)^{4}} \bar{\Psi}_{(+)}(p) \mathscr{S}_{(+)}^{-1}(p) \Psi_{(+)}(p)+\frac{1}{2} \int \frac{d^{4} p}{(2 \pi)^{4}} \bar{\Psi}_{(-)}(p) \mathscr{S}_{(-)}^{-1}(p) \Psi_{(-)}(p),
\end{array}
$$

where

$$
\begin{aligned}
& \mathscr{S}_{(0)}^{-1}(p)=\left(\begin{array}{cc}
{\left[G_{(0) 0}^{+}\right]^{-1}(p)} & \Delta_{(0)}^{-} \\
\Delta_{(0)}^{+} & {\left[G_{(0) 0}^{-}\right]^{-1}(p)}
\end{array}\right), \\
& \mathscr{S}_{(+)}^{-1}(p)=\left(\begin{array}{cc}
{\left[G_{(+) 0}^{+}\right]^{-1}(p)} & \Delta_{(+)}^{-} \\
\Delta_{(+)}^{+} & {\left[G_{(+) 0}^{-}\right]^{-1}(p)}
\end{array}\right),
\end{aligned}
$$




$$
\mathscr{S}_{(-)}^{-1}(p)=\left(\begin{array}{cc}
{\left[G_{(-) 0}^{+}\right]^{-1}(p)} & \Delta_{(-)}^{-} \\
\Delta_{(-)}^{+} & {\left[G_{(-) 0}^{-}\right]^{-1}(p)}
\end{array}\right)
$$

with

$$
\begin{gathered}
\Delta_{(+)}^{+}=\Omega_{-} \Delta^{+} \Omega_{+}, \\
\Delta_{(-)}^{+}=\Omega_{+} \Delta^{+} \Omega_{-}, \\
\Delta_{(0)}^{+}=\Omega_{0} \Delta^{+} \Omega_{0},
\end{gathered}
$$

Notice that to form the positive (negative) Nambu-Gorkov field we used the positive (negative) fermion field and the charge conjugate of the negative (positive) field. This is done so that the rotated charge of the up and down components in a given Nambu-Gorkov field be the same. This way to form the Nambu-Gorkov fields is mandated by what kind of field enters in a given condensate term, which in turn is related to the neutrality of the fermion condensate $\left\langle\bar{\psi}_{C} \psi\right\rangle$ with respect to the rotated $\tilde{Q}$-charge.

In momentum space the bare inverse propagator for the neutral field is

$$
\left[G_{(0) 0}^{ \pm}\right]^{-1}(p)=\left[\gamma_{\mu}\left(p_{\mu} \pm \mu \delta_{\mu 0}\right)-m\right]
$$

where the momentum is the usual $p=\left(p_{0}, p_{1}, p_{2}, p_{3}\right)$ of the case with no background field.

For positively and negatively charged fields the bare inverse propagators are

$$
\left[G_{(+) 0}^{ \pm}\right]^{-1}(p)=\left[\gamma_{\mu}\left(\bar{p}_{\mu}^{(+)} \pm \mu \delta_{\mu 0}\right)-m\right],
$$

and

$$
\left[G_{(-) 0}^{ \pm}\right]^{-1}(p)=\left[\gamma_{\mu}\left(\bar{p}_{\mu}^{(-)} \pm \mu \delta_{\mu 0}\right)-m\right]
$$

respectively.

\section{Gap Solutions}

The main question we would like to address now is: Can we find a region of magnetic fields where the gaps $\Delta_{A}$ and $\Delta_{A}^{B}$, (or $\Delta_{S}$ and $\Delta_{S}^{B}$ ), differ enough from each other that the system is not in the CFL phase anymore, but in the MCFL phase? To explore the possible answer to this question we need to solve the gap equations derived from the Nambu-Gorkov effective action (3.28).

In coordinate space the QCD gap equation reads

$$
\Delta^{+}(x, y)=i \frac{g^{2}}{4} \lambda_{A}^{T} \gamma^{\mu} S_{21}(x, y) \gamma^{v} \lambda_{B} D_{\mu v}^{A B}(x, y),
$$

where $S_{21}(x, y)$ is the off-diagonal part of the Nambu-Gorkov fermion propagator in coordinate space and, for simplicity, we have omitted explicit color and flavor indices in the gap and fermion propagator. Here $D_{\mu \nu}^{A B}$ is the gluon propagator. 
In a NJL model the gap equation can be obtained from Eq. (4.1) simply by substituting the gluon propagator by

$$
D_{\mu \nu}^{A B}(x, y)=\frac{1}{\Lambda^{2}} g_{\mu \nu} \delta^{A B} \delta^{(4)}(x-y) .
$$

The NJL model is characterized by a coupling constant $g$ and an ultraviolet cutoff $\Lambda$. The ultraviolet cutoff should be much larger than any of the energy scales of the system, typically the chemical potential. In the presence of a magnetic field we should also assume that $\Lambda$ is larger than the magnetic energy $\sqrt{\tilde{e} \tilde{B}}$. In other studies of color superconductivity within the NJL model, the values of $g$ and $\Lambda$ are chosen to match some QCD vacuum properties, thus hoping to get in such a way correct approximated quantitative results of the gaps. We will follow the same philosophy here, noticing however that this completely ignores the effect of the magnetic field on the gluon dynamics.

To solve the gap equation (4.1) for the whole range of magnetic-field strengths we need to use numerical methods. We have found, however, a situation where an analytical solution is possible. This corresponds to the case $\widetilde{e} \widetilde{B} \gtrsim \mu^{2} / 2$. Taking into account that the leading contribution to the gap solution comes from quark energies near the Fermi level, it follows that for fields in this range only the LLL $(l=0)$ contributes.

Using the approximation $\Delta_{A}^{B} \gg \Delta_{S}^{B}, \Delta_{A}$, and $\Delta_{A} \gg \Delta_{S}$, the gap equations decouple and the equation for $\Delta_{A}^{B}$ is

$$
\begin{aligned}
\Delta_{A}^{B} & \approx \frac{g^{2}}{3 \Lambda^{2}} \int_{\Lambda} \frac{d^{3} q}{(2 \pi)^{3}} \frac{\Delta_{A}^{B}}{\sqrt{(q-\mu)^{2}+2\left(\Delta_{A}^{B}\right)^{2}}} \\
& +\frac{g^{2} \widetilde{e} \widetilde{B}}{3 \Lambda^{2}} \int_{-\Lambda}^{\Lambda} \frac{d q}{(2 \pi)^{2}} \frac{\Delta_{A}^{B}}{\sqrt{(q-\mu)^{2}+\left(\Delta_{A}^{B}\right)^{2}}}
\end{aligned}
$$

where the first/second term in the r.h.s. of Eq.(4.3) corresponds to the contribution of $\widetilde{Q}$-neutral/charged quark propagators, respectively. For the last one, we dropped all Landau levels but the lowest, as we are interested in the leading term.

The solution of Eq. (4.3) reads

$$
\Delta_{A}^{B} \sim 2 \sqrt{\delta \mu} \exp \left(-\frac{3 \Lambda^{2} \pi^{2}}{g^{2}\left(\mu^{2}+\widetilde{e} \widetilde{B}\right)}\right),
$$

with $\delta \equiv \Lambda-\mu$. It can be compared with the antisymmetric CFL gap [13]

$$
\Delta_{A}^{\mathrm{CFL}} \sim 2 \sqrt{\delta \mu} \exp \left(-\frac{3 \Lambda^{2} \pi^{2}}{2 g^{2} \mu^{2}}\right) .
$$

In this approximation the remaining gap equations read

$$
\begin{aligned}
\Delta_{S}^{B} & \approx-\frac{g^{2}}{6 \Lambda^{2}} \int_{\Lambda} \frac{d^{3} q}{(2 \pi)^{3}} \frac{\Delta_{A}^{B}}{\sqrt{(q-\mu)^{2}+2\left(\Delta_{A}^{B}\right)^{2}}} \\
& +\frac{g^{2} \widetilde{e} \widetilde{B}}{6 \Lambda^{2}} \int_{-\Lambda}^{\Lambda} \frac{d q}{(2 \pi)^{2}} \frac{\Delta_{A}^{B}}{\sqrt{(q-\mu)^{2}+\left(\Delta_{A}^{B}\right)^{2}}}
\end{aligned}
$$




$$
\begin{aligned}
\Delta_{A} & \approx \frac{g^{2}}{4 \Lambda^{2}} \int_{\Lambda} \frac{d^{3} q}{(2 \pi)^{3}}\left(\frac{17}{9} \frac{\Delta_{A}}{\sqrt{(q-\mu)^{2}+\Delta_{A}^{2}}}\right. \\
& \left.+\frac{7}{9} \frac{\Delta_{A}}{\sqrt{(q-\mu)^{2}+2\left(\Delta_{A}^{B}\right)^{2}}}\right),
\end{aligned}
$$

and

$$
\begin{aligned}
\Delta_{S} & \approx \frac{g^{2}}{18 \Lambda^{2}} \int_{\Lambda} \frac{d^{3} q}{(2 \pi)^{3}}\left(\frac{\Delta_{A}}{\sqrt{(q-\mu)^{2}+\Delta_{A}^{2}}}\right. \\
& \left.-\frac{\Delta_{A}}{\sqrt{(q-\mu)^{2}+2\left(\Delta_{A}^{B}\right)^{2}}}\right) .
\end{aligned}
$$

We express below the solution of these gap equations as ratios over the CFL antisymmetric and symmetric gaps

$$
\frac{\Delta_{A}}{\Delta_{A}^{\mathrm{CFL}}} \sim \frac{1}{2^{(7 / 34)}} \exp \left(-\frac{36}{17 x}+\frac{21}{17} \frac{1}{x(1+y)}+\frac{3}{2 x}\right),
$$

where $x \equiv g^{2} \mu^{2} / \Lambda^{2} \pi^{2}$, and $y \equiv \widetilde{e} \widetilde{B} / \mu^{2}$, and

$$
\begin{aligned}
& \frac{\Delta_{S}^{B}}{\Delta_{S}^{\mathrm{CFL}}} \sim \frac{\Delta_{A}^{B}}{\Delta_{A}^{\mathrm{CFL}}}\left(\frac{3}{4}+\frac{9}{2 x \ln 2} \frac{y-1}{y+1}\right), \\
& \frac{\Delta_{S}}{\Delta_{S}^{\mathrm{CFL}}} \sim \frac{\Delta_{A}}{\Delta_{A}^{\mathrm{CFL}}} \frac{3}{2}\left(1-\frac{4}{1+y}\right) .
\end{aligned}
$$

Note that our analytic solutions are only valid at strong magnetic fields. The lower value $\widetilde{e} \widetilde{B} \sim \mu^{2} / 2$ corresponds to $\widetilde{e} \widetilde{B} \sim(0.8-1.1) \cdot 10^{18} \mathrm{G}$, for $\mu \sim 350-400 \mathrm{MeV}$. For fields of this order and larger the $\Delta_{A}^{B}$ gap is larger than $\Delta_{A}^{\mathrm{CFL}}$ at the same density values. Nevertheless, we have estimated (see the details in Cristina Manuel's talk in the proceedings) that the separation between CFL and MCFL will take place already at fields $\sim 10{ }^{16} \mathrm{G}$.

All the gaps feel the presence of the external magnetic field. The effect of the magnetic field in $\Delta_{A}^{B}$ is to increase the density of states, which enters in the argument of the exponential as typical of a BCS solution. The density of states appearing in (4.4) is just the sum of those of neutral and charged particles participating in the given gap equation (for each Landau level, the density of states around the Fermi surface for a charged quark is $\widetilde{e} \widetilde{B} / 2 \pi^{2}$ ).

All the $\widetilde{Q}$-charged quarks have common gap $\Delta_{\widetilde{A}}^{B}$. Hence, the densities of the charged quarks are all equal. As two of these quarks have positive $\widetilde{Q}$ charge, while the other two have it negative, the $\widetilde{Q}$ neutrality of the medium is guaranteed without having to introduce any electron density.

\section{Conclusions}

In this paper, we have shown that a magnetic field leads to the formation of a new color-flavor locking phase, characterized by a smaller vector symmetry than the CFL phase. The essential 
role of the penetrating magnetic field is to modify the density of states of charged quarks on the Fermi surface. To better understand the relevance of this new phase in astrophysics we need to explore the region of moderately strong magnetic fields $\widetilde{e} \widetilde{B}<\mu^{2} / 2$, which requires to carry out a numerical study of the gap equations including the effect of higher Landau levels. Because the total density of states around the Fermi surface for charged particles does not vary monotonically with the number of Landau levels, we still expect to find a meaningful splitting of the gaps at these fields and therefore a qualitative separation between the CFL and MCFL phases.

\section{Acknowledgments}

The work of E.J.F. and V.I. was supported in part by NSF grant PHY-0070986, and C.M. was supported by MEC under grant FPA2004-00996.

\section{References}

[1] M. Alford , K. Rajagopal and F. Wilczek, "Color Flavor Locking and Chiral Symmetry Breaking ," Nucl. Phys. B 537, 443 (1999).

[2] C. Thompson and R. C. Duncan, "The Soft Gamma Repeaters as Very Strongly Magnetized Neutron Stars. 2. Quiescent Neutrino, X-ray, and Alfven Wave Emission,” Astrophys. J. 473, 322 (1996).

[3] German Lugones, "Magnetic Fields in High-Density Stellar Matter, " [ast ro-ph / 0504454 ].

[4] L. Dong and S.L. Shapiro, "Cold equation of state in a strong magnetic field - Effects of inverse beta-decay," Astrophys. J. 383, 745 (1991).

[5] E. J. Ferrer, V. de la Incera and C. Manuel, "Magnetic Color Flavor Locking Phase in High Density QCD,” PRL 95, 152002 (2005).

[6] E. V. Gorbar,"Color superconductivity in an external magnetic field," Phys. Rev. D 62, 014007 (2000); K. Iida and G. Baym, "Superfluid phases of quark matter. III. Supercurrents and vortices," Phys. Rev. D 66, 014015 (2002); I. Giannakis and H-C Ren, "The Ginzburg-Landau theory and the surface energy of a colour superconductor," Nucl. Phys. B 669, 462 (2003).

[7] Alford M, Berges J, and Rajagopal K, "Magnetic fields within color superconducting neutron star cores," Nucl. Phys. B 571, 269 (2000).

[8] V. A. Miransky, and I. A. Shovkovy, "Magnetic catalysis and anisotropic confinement in QCD," Phys. Rev. D 66, 045006 (2002).

[9] V.I. Ritus, "Radiative Corrections in Quantum Elctrodynamics with Intense Field and their Analytical Properties," Ann.Phys. 69, 555 (1972).

[10] E. Elizalde, E. J. Ferrer, and V. de la Incera, "Neutrino Self-Energy and Index of Refraction in Strong Magnetic Field: A New Approach," Ann. of Phys. 295, 33 (2002); "Neutrino Propagation in a Strongly Magnetized Medium," Phys. Rev. D 70, 043012 (2004).

[11] C. N. Leung, and S.-Y. Wang, "Gauge independent approach to chiral symmetry breaking in a strong magnetic field," hep-ph/0510066.

[12] D.-S Lee, C. N. Leung and Y. J. Ng, "Chiral symmetry breaking in a uniform external magnetic field," Phys. Rev. D 55, 6504 (1997); E. J. Ferrer, and V. de la Incera, "Ward-Takahashi Identity with External Field in Ladder QED," Phys. Rev. D 58, 065008 (1998); "Magnetic Catalysis in the Presence of Scalar Fields Phys. Lett. B 481, 287 (2000); E. Elizalde, E. J. Ferrer, and V. de la Incera, "Beyond-Constant-Mass-Approximation Magnetic Catalysis in the Gauge Higgs-Yukawa Model," Phys. Rev. D 68, 096004 (2003). 
[13] K. Rajagopal and F. Wilczek, "The condensed matter physics of QCD, " hep-ph/0011333. 